\title{
Article \\ Theory of Photon Subtraction for Two-Mode Entangled Light Beams
}

\author{
Oscar Rosas-Ortiz ${ }^{1, *(D)}$ and Kevin Zelaya ${ }^{2}$ (D) \\ 1 Physics Department, Cinvestav, AP 14-740, Mexico City 07000, Mexico \\ 2 Nuclear Physics Institute, The Czech Academy of Science, Řež, 25068 Husinec, Czech Republic; \\ zelayame@crm.umontreal.ca \\ * Correspondence: orosas@fis.cinvestav.mx
}

Citation: Rosas-Ortiz, O.; Zelaya, K. Theory of Photon Subtraction for Two-Mode Entangled Light Beams. Quantum Rep. 2021, 3, 500-516. https://doi.org/10.3390/ quantum 3030033

Academic Editor: Margarita A. Man'ko

Received: 19 July 2021

Accepted: 27 August 2021

Published: 3 September 2021

Publisher's Note: MDPI stays neutral with regard to jurisdictional claims in published maps and institutional affiliations.

Copyright: (c) 2021 by the authors. Licensee MDPI, Basel, Switzerland. This article is an open access article distributed under the terms and conditions of the Creative Commons Attribution (CC BY) license (https:/ / creativecommons.org/licenses/by/ $4.0 /)$.

\begin{abstract}
Photon subtraction is useful to produce nonclassical states of light addressed to applications in photonic quantum technologies. After a very accelerated development, this technique makes possible obtaining either single photons or optical cats on demand. However, it lacks theoretical formulation enabling precise predictions for the produced fields. Based on the representation generated by the two-mode $S U(2)$ coherent states, we introduce a model of entangled light beams leading to the subtraction of photons in one of the modes, conditioned to the detection of any photon in the other mode. We show that photon subtraction does not produce nonclassical fields from classical fields. It is also derived a compact expression for the output field from which the calculation of conditional probabilities is straightforward for any input state. Examples include the analysis of squeezed-vacuum and odd-squeezed states. We also show that injecting optical cats into a beam splitter gives rise to entangled states in the Bell representation.
\end{abstract}

Keywords: nonclassical states of light; photon subtraction; SU(2) coherent states; squeezed-vacuum; odd-squeezed states; optical Schrödinger cats

\section{Introduction}

Nonclassical states of light are very useful to develop photonic quantum technologies [1]. Considerable attention has been devoted to photon-number states $|n\rangle$ since they permit the realization of quantum communication in several forms [2]: 1 photon to send messages, 2 photons to prepare a given quantum state at a distance, 3 photons to teleport quantum states, and so on. Quite remarkably, scalable quantum computing is possible by using single photon sources [3]. In the same context, as the squeezed states of light have less noise in one of their quadratures than the quantum noise limit dictates [4], they are useful to improve the precision of interferometric measurements dealing with very low intensity light signals $[5,6]$. The prototypical example is the squeezed-vacuum state, which consists entirely of even-photon number states [7]. The counterpart of squeezed-vacuum, called odd-squeezed state, includes odd-photon number states only [8]. Additionally, the even and odd coherent states [9] are constructed as opposite phase superpositions of the fully coherent states introduced by Glauber [10]. This issue is of great interest because the Glauber states, formed by superpositions of photon-number states, tolerate a description in terms of the Maxwell theory [5], so they may describe the states of macroscopic systems. The creation of cat states, a theoretical description introduced by Schrödinger to show the way in which quantum mechanics contradicts our everyday experience for systems as great as a 'cat' [11], therefore, is available in the laboratory using even and odd coherent states [12,13], which are, therefore, called optical cat states [14] (although the term optical kitten is also found [15]).

Optical cat states can be experimentally prepared by photon subtraction from a squeezed vacuum state [15-21] and find immediate applications in diverse photonic quantum technologies, including quantum information [22] and quantum teleportation [23]. 
Their nonclassical properties have been analyzed in terms of the sub-Poissonian statistics and the negativity of the Wigner function $[24,25]$, where it has been shown that singlephoton subtracted squeezed states decay to vacuum [25]. The optical cat states are also instrumental in the study of entanglement [26-29].

Photon subtraction offers a practical way of getting single photons on demand from weakly squeezed vacuum $[15,17,19,20,30,31]$. The first antecedents can be traced back to the study of two-mode electromagnetic fields expressed as superpositions of $S U(2)$ coherent states [32], including the development of quantum lithography [33,34], where the occurrence of NOON states [35] is quite natural [36]. The primordial NOON state, with $N=2$ is the result of the Hong-Ou-Mandel effect [37,38]. The production of NOON states for higher values of $\mathrm{N}$ was proposed in reference [36] via conditioned photo detection. Experimental success for $N \leq 5$ has been reported by using different techniques in, e.g., references [39-42].

A very practical version of the photon subtraction technique considers beam splitters to generate two-mode entangled light beams. The process changes the quantum statistics of the input fields so that the output modes are correlated in nonclassical form. Therefore, the field in one of the output modes is conditioned to the result of measuring a given number of photons in the other mode. Formally, lossless beam splitters are associated with the symmetries of the $S U(2)$ Lie group [43]. Indeed, the $S U(2)$ coherent states [44] may be represented in two-mode form [32,45-47], so they coincide with the output of a 50/50 beamsplitter that is injected with $n$-photons in one input channel and $m$-photons in the other input channel [48]. It has been shown that, when the two input modes have the same number of photons, the output state includes a superposition of even photon-number states only [48], so the possibility of having odd numbers of photons is zero [49]. Besides, the entanglement properties of the output fields are strengthened if the input modes contain different number of photons [48]. The latter is usually considered by injecting a superposition of photon-number states into one of the input channels and vacuum in the other one [25].

In this work, we provide a theoretical approach for photon subtraction in two-mode entangled light beams. The model considers a lossless symmetric beam splitter to generate nonclassical correlations between the output modes. The main idea is to represent the output fields as linear superpositions of $S U(2)$ coherent states that exhibit nonclassical properties. We, therefore, find the conditions for non-separability in the output fields, which are intimately connected with the quantum properties of the input state. In particular, we show that photon subtraction does not produce nonclassical fields from classical fields. We derive a compact expression for the output field from which the calculation of conditional probabilities is straightforward. Our purpose is aimed at making up for the lack of theoretical formulations of the photon subtraction technique, which has been developed mainly in experimental form.

The structure of the paper is as follows. In Section 2, we revisit the two-mode representation based on the $S U(2)$ coherent states and discuss the generalities of the two-mode states that can be constructed in terms of such basis. In Section 3, we introduce the structure of photon-subtracted states associated with any input field consisting of photon-number state superpositions. We discuss about the conditions for photon subtraction and derive a compact expression for the two-mode entangled fields produced by a beam splitter. In Section 4 , we show the applicability of our model generating photon-subtracted versions of the squeezed-vacuum and the odd-photon squeezed states. We also study the result of injecting optical cat states into the beam splitter, which leads to entangled states in the form of the Bell-basis elements. A short appendix includes calculations that are useful to reproduce the results of the main text.

\section{Two-Mode Entangled States}

The states of two-mode light beams are elements of the separable Hilbert space $\mathcal{H}=\operatorname{span}\{|n, m\rangle, n, m=0,1, \ldots\}$, where the bipartite states $|n\rangle_{a} \otimes|m\rangle_{b}=|n, m\rangle$ are 
orthonormal, with $|n\rangle_{a}$ and $|m\rangle_{b}$ forming the orthonormal bases of the space of photonnumber states for modes $a$ and $b$, respectively. A given element $|\varphi\rangle \in \mathcal{H}$ is separable if there exists a pair of vectors $|\phi\rangle_{a}$ and $|\theta\rangle_{b}$ such that $|\varphi\rangle=|\phi\rangle_{a} \otimes|\theta\rangle_{b}$. Otherwise, $|\varphi\rangle$ is said to be non-separable or entangled.

Next, we provide a formulation to construct a new basis for $\mathcal{H}$ that is expressed as non-separable combinations of $|n, m\rangle$. The main interest is to facilitate the construction and analysis of two-mode entangled states in $\mathcal{H}$, such that they describe the outputs of a beam splitter and the basic ingredient for photon subtraction, as well.

\subsection{Bipartite Space of States}

The $S U(2)$ coherent states [44] can be constructed in the two mode Hilbert space $\mathcal{H}$, the representation of which is obtained by applying the unitary operator,

$$
\hat{U}(\xi)=\exp \left[\xi \hat{a}^{\dagger} \hat{b}-\xi^{*} \hat{a} \hat{b}^{\dagger}\right], \quad \xi=|\xi| e^{i \varphi}, \quad \varphi \in[-\pi, \pi),
$$

to the bipartite state $|n, 0\rangle$, where $\hat{a}, \hat{a}^{\dagger}$, and $\hat{b}, \hat{b}^{\dagger}$, are the boson ladder operators for modes $a$ and $b$, respectively [32,46,47].

The operator $\hat{U}(\xi)$ may be used to represent a lossless symmetric beam splitter, with amplitudes of transmission and reflection $t=\cos |\xi|$ and $r=\sin |\xi|$ [43]. The parameter $\varphi$ denotes a phase difference between the transmitted and reflected beams. Then, $\hat{U}(\xi)|n, m\rangle$ is the result of injecting $n$ photons into channel $a$ and $m$ photons into channel $b$ of the beam splitter. The output state $\hat{U}(\xi)|n, m\rangle$ exhibits entanglement properties that can be strengthened if $n \neq m$ [48], which is precisely the case for the two-mode $S U(2)$ coherent states $\hat{U}(\xi)|n, 0\rangle$.

In this paper, we consider an idealized $50 / 50$ beam splitter $\hat{B}$, represented by $\hat{U}(\tilde{\xi})$ with $\xi=i \frac{\pi}{4}$. That is:

$$
\hat{B}=\exp \left[i \frac{\pi}{4}\left(a^{\dagger} b+a b^{\dagger}\right)\right] .
$$

The corresponding two-mode $S U(2)$ coherent states acquire the form [48]

$$
|n, 0\rangle_{B}=\hat{B}|n, 0\rangle=\sum_{k=0}^{n} c_{k, n-k}|k, n-k\rangle, \quad c_{k, n-k}=\frac{1}{\sqrt{2^{n}}}\left(\begin{array}{c}
n \\
k
\end{array}\right)^{1 / 2} e^{i \frac{\pi}{2} k} .
$$

The set $|n, 0\rangle_{B}$ is orthonormal, so it defines a concrete representation in the two-mode Hilbert space $\mathcal{H}$; the first elements are provided in Equation (A1) of Appendix A. The change of basis between the sets $|n, 0\rangle_{B}$ and $|s, m\rangle$ is ruled by the unitary operator (2). The advantage of working in the representation $|n, 0\rangle_{B}$ is that the basis elements are nonseparable when they are expressed in representation $|s, m\rangle$. The latter means that $|n, 0\rangle_{B}$ encodes nonclassical correlations between mode $a$ and mode $b$, with exception of $|0,0\rangle_{B}$.

As the result of injecting $n$-photons in channel $a$ of the beam splitter $\hat{B}$, and 0 -photons in channel $b$, the basis $|n, 0\rangle_{B}$ shows very useful properties. In particular, finding $m$ photons in mode $a$ implies $r=n-m$ photons in mode $b$. Thus, for any state (3), we have the conditional probability

$$
\mathcal{P}_{m, r}=\frac{1}{2^{n}}\left(\begin{array}{c}
n \\
k
\end{array}\right)=\frac{\Gamma\left(r+\frac{1}{2}\right) \Gamma\left(m+\frac{1}{2}\right)}{\Gamma(r+1) \Gamma(m+1)}\left[\frac{1}{2^{r+m} B\left(r+\frac{1}{2}, m+\frac{1}{2}\right)}\right], \quad n=m+r,
$$

where $B(x, y)=\frac{\Gamma(x) \Gamma(y)}{\Gamma(x+y)}$ is the Euler beta function [50], which cannot be factorized as $B(x, y)=f(x) g(y)$ for any functions $f$ and $g$. Therefore, $\mathcal{P}_{m, r} \neq \mathcal{P}_{m} \mathcal{P}_{r}$, with $\mathcal{P}_{m}$ and $\mathcal{P}_{r}$ two independent probability distributions, one for each output port of the beam splitter. This property is concomitant to the impossibility of writing the $S U(2)$ coherent states (3) as the product of two independent mode states. That is, if $n \neq m$, then $\mathcal{P}_{m, r} \neq \mathcal{P}_{m} \mathcal{P}_{r}$ implies $|n, 0\rangle_{B} \neq|\phi\rangle_{a} \otimes|\theta\rangle_{b}$ [51]. The latter is a direct consequence of the nonclassical correlations between modes $a$ and $b$ that are encoded in states $|n, 0\rangle_{B}$. 


\subsection{Representations in the Bipartite State Space}

For any regular vector $|\Psi\rangle \in \mathcal{H}$, one has

$$
|\Psi\rangle_{B}=\frac{1}{\lambda} \sum_{n=0}^{\infty} \alpha_{n}|n, 0\rangle_{B}=\frac{1}{\lambda} \sum_{n=0}^{\infty} \sum_{k=0}^{n} \alpha_{n} c_{k, n-k}|k, n-k\rangle, \quad \alpha_{n} \in \mathbb{C},
$$

where $\lambda \in \mathbb{C}$ stands for normalization. Noticeably, we may also write

$$
|\Psi\rangle_{B}=\hat{B}|\psi(\alpha), 0\rangle,
$$

with

$$
|\psi(\alpha)\rangle_{a}=\frac{1}{\lambda(\alpha)} \sum_{n=0}^{\infty} \alpha_{n}|n\rangle_{a},
$$

a normalized linear combination of number states in the $a$ mode. That is, we can construct regular two-mode states $|\Psi\rangle_{B}$ by injecting regular superpositions $|\psi(\alpha)\rangle_{a}$ into the $a$-port of the beam splitter $\hat{B}$.

For the sake of simplicity, in Equations (6) and (7), we have introduced the shortcut notation $|\psi(\alpha)\rangle:=\left|\psi\left(\alpha_{1}, \alpha_{2}, \ldots\right)\right\rangle$. Consistently, $\lambda(\alpha):=\lambda\left(\alpha_{1}, \alpha_{2}, \ldots\right)$. This notation is adopted for similar expressions throughout the manuscript.

The quantum properties of state $|\Psi\rangle_{B}$ depend on the combined amplitude probabilities $\alpha_{n} c_{k, n-k}$, which may lead to either separable (classical) or non-separable (nonclassical) states in $\mathcal{H}$; see the discussion of Equation (4) above. That is, although the two-mode basis elements $|n, 0\rangle_{B}$ encode entanglement between modes $a$ and $b$ (with exception of $\left.|0,0\rangle_{B}\right)$, their linear superpositions $|\Psi\rangle_{B}$ may lack such entanglement. This property is very common in quantum physics [5], with the coherent states $|z\rangle$ of Glauber [10] as a prototypical example. Indeed, decomposing $|z\rangle$ into a pair of superpositions, one consisting entirely of even-photon number states and the other including odd-photon number states only, one arrives at the even and odd coherent states of Dodonov, Malkin, and Man'ko [9], which are nonclassical [52]. Hence, the fully coherent (classical) state $|z\rangle$ is a superposition of two nonclassical states, while the even and odd coherent states are superpositions of two classical states!

We are mainly interested in nonclassical two-mode states $|\Psi\rangle_{B}$, since the quantum correlations between modes $a$ and $b$ are fundamental to construct photon-subtracted states successfully.

\section{Conditions for Photon Subtraction}

The straightforward calculation shows that the two-mode state (5) can be rewritten as follows:

$$
|\Psi\rangle_{B}=\frac{1}{\lambda} \sum_{n=0}^{\infty} \frac{e^{i \frac{\pi}{2} n}}{\sqrt{n !}} \lambda_{n}(\beta)|n\rangle_{a} \otimes\left|\psi_{n}(\beta)\right\rangle_{b},
$$

where $\left|\psi_{n}(\beta)\right\rangle$ and $\lambda_{n}(\beta)$ are written in the shortcut notation introduced above, with $\beta_{n}=\frac{\alpha_{n}}{\sqrt{2^{n}}}$, and

$$
\left|\psi_{n}(\alpha)\right\rangle_{a}=\frac{1}{\lambda_{n}(\alpha)} \sum_{k=0}^{\infty} \sqrt{\frac{(k+n) !}{k !}} \alpha_{k+n}|k\rangle_{a} ;
$$

see details in Appendix A.

The vector $\left|\psi_{n}(\alpha)\right\rangle_{a}$ results of applying $n$-times the annihilator operator $\hat{a}$ on the input state (7), so it represents the subtraction of $n$ photons from $|\psi(\alpha)\rangle_{a}$. Hereafter, this vector will be referred to as the $n$-subtracted state of $|\psi(\alpha)\rangle_{a}$. 
As $\left|\psi_{n}(\beta)\right\rangle_{b}$ in (8) is the $b$ mode version of (9), evaluated with the reduced probability amplitudes $\beta_{n}=\alpha_{n} / \sqrt{2^{n}}$, and the beam splitter $\hat{B}$ is lossless and symmetric, the output (8) can be also written in the form

$$
|\Psi\rangle_{B}=\frac{1}{\lambda} \sum_{k=0}^{\infty} \frac{e^{-i \frac{\pi}{2} k}}{\sqrt{k !}} \lambda_{k}(\beta)\left|\psi_{k}(\beta)\right\rangle_{a} \otimes|k\rangle_{b} .
$$

Thus, detecting a given number of photons in any of the two output ports, the beam in the other port is represented by a subtracted photon state.

In the sequel, we concentrate in representation (8). The output ports $a$ and $b$ of $\hat{B}$ will be referred to as idler and signal. Consistently, the modes $a$ and $b$ in $|\Psi\rangle_{B}$ will be, respectively, called idler and signal. Besides, to generate subtracted photon states in the signal channel, we assume that idealized photodetectors with unit efficiency are used to collect photons at the idler channel.

Depending on $\alpha_{n}$, the two-mode state (8) will exhibit nonclassical correlations between modes $a$ and $b$. The photon subtraction operates whenever $|\Psi\rangle_{B}$ is not factorized as the product of two independent states, one for mode $a$ and one for mode $b$. Thus, entanglement between idler and signal output channels is necessary to link the occurrence of the nsubtracted signal state $\left|\psi_{n}(\beta)\right\rangle_{b}$ with the detection of exactly $n$-idler photons.

According to the conjecture that entangled output states from a beam splitter require nonclassicality in the input port [48], the first clue to produce photon subtraction is to consider nonclassical states $|\psi(\alpha)\rangle_{a}$. Another trail is reached by noticing that a very special class of probability amplitudes fulfilling

$$
\alpha_{k+n}=\frac{1}{\sqrt{(k+n) !}} \delta_{k} \gamma_{n}
$$

leads to separable summations in (8), and then to separable versions of the output state. Indeed, introducing (10) into the pair of Equations (9) and (8) yields

$$
|\Psi\rangle_{B}=\frac{\lambda(\widetilde{\delta}) \lambda(\widetilde{\gamma})}{\lambda}|\psi(\widetilde{\gamma})\rangle_{a} \otimes|\psi(\widetilde{\delta})\rangle_{b}, \quad \widetilde{\gamma}_{n}=\frac{e^{i \frac{\pi}{2} n} \gamma_{n}}{\sqrt{2^{n}}}, \quad \widetilde{\delta}_{k}=\frac{\delta_{k}}{\sqrt{2^{k}}}
$$

The output field (11) is separable, so it does not encode nonclassical correlations between modes $a$ and $b$. Besides, following reference [48], the input field constructed with the probability amplitudes (10) is classical by necessity. Thus, we have shown that photon subtraction of classical fields does not produce nonclassical fields.

Probability amplitudes fulfilling (10) give rise to independent probabilities for detecting photons in either of the output channels. They are intimately connected with the separability of bipartite states [51], so their identification is complementary to the conjecture introduced in reference [48].

\subsection{Classical Correlations}

The simplest form to obtain factorized states (8) involves scalable probability amplitudes, such as $\alpha_{n}=\frac{z^{n}}{\sqrt{n !}}$, since (10) is fulfilled with $\gamma_{n}=z^{n}$ and $\delta_{k}=z^{k}$. In this case, the input (7) is a Glauber state [10]:

$$
|z\rangle=\frac{1}{\lambda_{G}} \sum_{n=0}^{\infty} \alpha_{n}^{G}|n\rangle, \quad \lambda_{G}(z)=e^{\frac{|z|^{2}}{2}}, \quad \alpha_{n}^{G}=\frac{z^{n}}{\sqrt{n !}} .
$$

Then, Equation (8) is reduced to the factorized form (11). Explicitly,

$$
|Z\rangle_{B}=\left|\frac{i z}{\sqrt{2}}\right\rangle_{a} \otimes\left|\frac{z}{\sqrt{2}}\right\rangle_{b}=\left|\frac{i z}{\sqrt{2}}, \frac{z}{\sqrt{2}}\right\rangle .
$$


Notably, the reduced probability amplitude $\beta_{n}$ coincides in form with $\alpha_{n}$, but changing the complex parameter $z$ by $\frac{1}{\sqrt{2}} z$. Recalling that the expected value for the number of photons $\hat{n}$ in a Glauber state is $\langle\hat{n}\rangle=|z|^{2}$, we see that the factors of $|Z\rangle_{B}$ are Glauber states with $\frac{1}{2}\langle\hat{n}\rangle$. The latter is consistent with the behavior of Gaussian laser beams that are injected into a beam splitter. For actual beam splitters $(\approx 50 / 50)$, the output is a pair of Gaussian beams with intensity that is approximately one half the input intensity. Moreover, detecting a photon in either of the output channels does not affect the nature of the field in the other channel. The above properties are explained by the fact that the output system does not include quantum correlations between their components. The result may be considered classical in two forms: On the one hand, the probability $\mathcal{P}_{n, m}$ of detecting $n$-idler photons and $m$-signal photons can be expressed as the product of two independent Poisson distributions with mean value $\frac{|z|^{2}}{2}$, which is a fingerprint of classicalness [51]. On the other hand, each of the output modes is a fully coherent state, so they are classical in the sense that tolerate a description in terms of the Maxwell theory [5]. Thus, the output state (13) verifies that the photon subtraction of classical fields does not produce nonclassical fields.

\subsection{Non-Classical Correlations}

Assuming that (8) is not separable, the number of idler photons will determine the $n$-subtracted signal state $\left|\psi_{n}(\beta)\right\rangle_{b}$. To be concrete, the expression

$$
\mathcal{P}_{n}(\alpha)=\left|\frac{\lambda_{n}(\beta)}{\lambda(\alpha)}\right|^{2} \frac{1}{n !}
$$

provides the probability of finding $n$ photons in the idler channel and state $\left|\psi_{n}(\beta)\right\rangle_{b}$ in the signal channel. The behavior of $\mathcal{P}_{n}(\alpha)$ depends on the concrete analytical expression of $\lambda_{n}(\beta)$, so the success of subtracting photons from the signal beam is markedly determined by the properties of the input state through the amplitude probabilities $\alpha_{n}$.

To facilitate applications, the following expressions provide the form acquired by (8) for input states $\left|\psi_{E}(\alpha)\right\rangle$ and $\left|\psi_{O}(\alpha)\right\rangle$, consisting entirely of even-photons and odd-photons in the superposition (7). Thus,

$$
\begin{aligned}
\left|\Psi_{E}\right\rangle_{B}=\frac{1}{\lambda_{E}} \sum_{n=0}^{\infty}\left[\frac{e^{i \pi n}}{\sqrt{(2 n) !}} \lambda_{2 n}^{E}(\beta)|2 n\rangle_{a} \otimes\left|\psi_{2 n}^{E}(\beta)\right\rangle_{b}\right. \\
\left.+\frac{i e^{i \pi n}}{\sqrt{(2 n+1) !}} \lambda_{2 n+1}^{E}(\beta)|2 n+1\rangle_{a} \otimes\left|\psi_{2 n+1}^{E}(\beta)\right\rangle_{b}\right],
\end{aligned}
$$

and

$$
\begin{aligned}
\left|\Psi_{O}\right\rangle_{B}=\frac{1}{\lambda_{O}} \sum_{n=0}^{\infty}\left[\frac{e^{i \pi n}}{\sqrt{(2 n) !}} \lambda_{2 n}^{O}(\beta)|2 n\rangle_{a} \otimes\left|\psi_{2 n}^{O}(\beta)\right\rangle_{b}\right. \\
\left.+\frac{i e^{i \pi n}}{\sqrt{(2 n+1) !}} \lambda_{2 n+1}^{O}(\beta)|2 n+1\rangle_{a} \otimes\left|\psi_{2 n+1}^{O}(\beta)\right\rangle_{b}\right]
\end{aligned}
$$

represent the result of injecting even states $\left|\psi_{E}(\alpha)\right\rangle$ and odd states $\left|\psi_{O}(\alpha)\right\rangle$ into the beam splitter, respectively. Concrete expressions for the $n$-subtracted states of $\left|\psi_{E}(\alpha)\right\rangle$ and $\left|\psi_{O}(\alpha)\right\rangle$ are given in Equations (A6), (A7), (A9) and (A10) of Appendix A.

\section{Applications}

Equation (8) comprises information that is necessary to describe the photon subtraction (7) of an input state $|\psi(\alpha)\rangle$. The related output $|\Psi\rangle_{B}$ is a linear superposition of entangled bipartite states whose probability amplitudes yield the probability (14). Each element in the superposition links a photon number state $|n\rangle_{a}$ of mode $a$ with the $n$-subtracted state (9) 
of $|\psi(\alpha)\rangle$ in mode $b$. Additionally, Equations (15) and (16) provide the above formulae assuming that the input state $|\psi(\alpha)\rangle$ is composited only of either even-number states or odd-number states, respectively. All these results are useful to further analyze properties, such as the mean value of dynamical variables, photon-statistics, and the negativity of the corresponding Wigner function.

Next, we apply our method to study photon subtraction in three different cases that are of interest.

\subsection{Squeezed-Vacuum State}

We consider the squeezed-vacuum state

$$
\left|\xi^{\mathrm{vac}}\right\rangle=\left(1-|\xi|^{2}\right)^{1 / 4} \sum_{n=0}^{\infty} \frac{\sqrt{(2 n) !}}{n !}\left(-\frac{\xi}{2}\right)^{n}|2 n\rangle, \quad|\xi|<1 .
$$

The beam splitter output $\left|\xi^{\mathrm{vac}}, 0\right\rangle_{B}$ is easily calculated from (15) and the $n$-subtracted states

$$
\begin{gathered}
\left|\xi_{2 n}^{\mathrm{vac}}\right\rangle=\frac{1}{\lambda_{2 n}^{\mathrm{vac}}(\xi)} \sum_{k=0}^{\infty} \frac{1}{\sqrt{(2 k) !}} \frac{(2 k+2 n) !}{(k+n) !}\left(-\frac{\xi}{2}\right)^{k+n}|2 k\rangle, \\
\left|\xi_{2 n+1}^{\mathrm{vac}}\right\rangle=\frac{1}{\lambda_{2 n+1}^{\mathrm{vac}}(\xi)} \sum_{k=0}^{\infty} \frac{1}{\sqrt{(2 k+1) !}} \frac{(2 k+2 n+2) !}{(k+n+1) !}\left(-\frac{\xi}{2}\right)^{k+n+1}|2 k+1\rangle .
\end{gathered}
$$

The normalizations are defined by the formulae

$$
\begin{array}{r}
\left|\lambda_{2 n}^{\mathrm{vac}}(\xi)\right|^{2}=\left(\frac{|\xi|}{2}\right)^{2 n}\left[\frac{\Gamma(2 n+1)}{\Gamma(n+1)}\right]_{2}^{2}{ }_{2}\left(n+\frac{1}{2}, n+\frac{1}{2}, \frac{1}{2} ;|\xi|^{2}\right), \\
\left|\lambda_{2 n+1}^{\mathrm{vac}}(\xi)\right|^{2}=\left(\frac{|\xi|}{2}\right)^{2 n+1}\left[\frac{\Gamma(2 n+3)}{\Gamma(n+2)}\right]_{2}^{2} F_{1}\left(n+\frac{3}{2}, n+\frac{3}{2}, \frac{3}{2} ;|\xi|^{2}\right) .
\end{array}
$$

The above expressions are in full agreement with the results already reported in reference [25], where special attention is payed to the 1-subtracted state $\left|\xi_{1}^{\text {vac }}\right\rangle$ derived from (19).

We would like to emphasize that, according to Equation (15), the two-mode state $\left|\xi^{\mathrm{vac}}, 0\right\rangle_{B}$ is expressed as a linear combination of $\left|\xi_{2 n}^{\mathrm{vac}}\right\rangle$ and $\left|\xi_{2 n+1}^{\mathrm{vac}}\right\rangle$, calculated in the $\beta$ configuration defined by the reduced probability amplitudes $\beta_{k}=\alpha_{k} / \sqrt{2^{k}}$, with $\alpha_{k}$ the probability amplitudes of (18) and (19), respectively. The straightforward calculation shows that $\left|\xi^{\mathrm{vac}}, 0\right\rangle_{B}$ is, therefore, parameterized by $\frac{\xi}{\sqrt{2}}$, with $\xi$ the complex-number characterizing the squeezed-vacuum state (17). Thus, even if no photons are detected at channel $a$, the signal field is different from the input beam (17). The latter is clear by recalling that $\xi$ characterizes predictions, such as the mean value of the number of photons. This phenomenon is explained by the entanglement of the basis elements $|n, 0\rangle_{B}$, which is preserved by the probability amplitudes defining Equation (17). That is, the idler vacuumstate $|0\rangle_{a}$ is also correlated with the signal field in nonclassical form. As a consequence, the input field is always affected when passing through the beam splitter.

In optics, it is well known that beam splitters reduce the intensity of light beams [12,13]. Indeed, as the transmission coefficient $|t|^{2}$ is always less than one, the field of the transmitted field is reduced by a factor $t$. Hence, "if observation is made only on the transmitted beam, the beam splitter is just an attenuator of the light beam" [12]. Consistently, detecting 0 -photons at the idler channel, we obtain an attenuated version $\left|\xi_{0}^{\mathrm{vac}}\right\rangle$ of the input field $\left|\xi^{\mathrm{vac}}\right\rangle$ in the signal mode.

As we are looking for subtraction of photons in the signal mode, detecting 0-photons in idler mode is the "no success" event, which, in turn, is the most probable. 
Figure 1 shows in dotted-blue the probability of finding $n$-subtracted states of $\left|\xi^{\text {vac }}\right\rangle$ in signal mode. As indicated above, the unsuccessful subtraction of photons $(n=0)$ is the highest possible result. In comparison, the probability of success $(n \geq 1)$ is drastically reduced, which justifies the technical difficulties to prepare photon-subtracted versions of the squeezed-vacuum in laboratory.

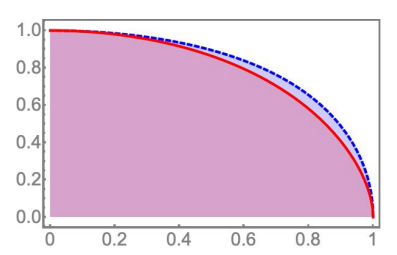

(a) 0-photon

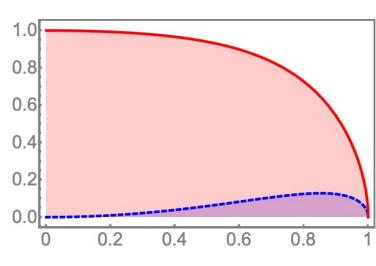

(b) 1-photon

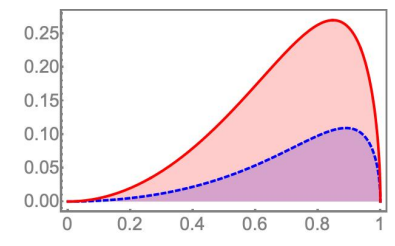

(c) 2-photon

Figure 1. Probability of photon subtraction for squeezed-vacuum $\left|\xi^{\mathrm{vac}}\right\rangle$ and odd-photon squeezed $\left|\xi^{\text {odd }}\right\rangle$ states, blue-dotted and red curves, respectively. The horizontal axis corresponds to $|\xi|$. In both cases, the probability of no success is very high, which justifies the technical difficulties to prepare photon-subtracted states in laboratory. In addition, in both cases, the probability of success decreases as the number of subtracted photons increases.

Interestingly, the above results may be managed to produce single photons on demand. Making $|\xi| \ll 1$, the input state $\left|\xi^{\text {vac }}\right\rangle$ can be expanded up to the first photon-number states as follows. Recall that $\left|\xi^{\text {vac }}\right\rangle$ results from the application of the squeezing operator

$$
\hat{S}(\xi)=\exp \left[\frac{\xi}{2} a^{\dagger 2}-\frac{\xi^{*}}{2} a^{2}\right]
$$

on the vacuum state $|0\rangle$. The series expansion yields

$$
\hat{S}(\xi)=\mathbb{I}+\frac{\xi}{2} a^{+2}-\frac{\xi^{*}}{2} a^{2}+\frac{1}{2}\left[\frac{\xi}{2} a^{+2}-\frac{\xi^{*}}{2} a^{2}\right]^{2}+\cdots .
$$

Then, up to first order in $|\xi|$, one gets $\left|\xi^{\mathrm{vac}}\right\rangle=\hat{S}(\xi)|0\rangle \approx|0\rangle+\frac{\xi}{\sqrt{2}}|2\rangle$. The action of the beam splitter on the latter approximate state gives

$$
\left|\xi^{\mathrm{vac}}, 0\right\rangle_{B} \approx|0,0\rangle_{B}+\frac{\xi}{\sqrt{2}}|2,0\rangle_{B}=|0,0\rangle+\frac{\xi}{\sqrt{2}}\left(\frac{1}{2}|0,2\rangle+\frac{i}{\sqrt{2}}|1,1\rangle-\frac{1}{2}|2,0\rangle\right) .
$$

So,

$$
\left|\xi^{\mathrm{vac}}, 0\right\rangle_{B} \approx|0\rangle_{a} \otimes\left(|0\rangle_{b}+\frac{\xi}{2 \sqrt{2}}|2\rangle_{b}\right)+i \frac{\xi}{2}|1\rangle_{a} \otimes|1\rangle_{b}-\frac{\xi}{2 \sqrt{2}}|2\rangle_{a} \otimes|0\rangle_{b}
$$

predicts the production of one single photon in channel $b$ by detecting one photon in channel $a$.

Figures 2 and 3 show, respectively, the photon distribution for the $n$-subtracted states $\left|\xi_{2 n+1}^{\mathrm{vac}}\right\rangle$ and $\left|\xi_{2 n}^{\mathrm{vac}}\right\rangle$, with $|\xi| \ll 1$. In both cases, it is privileged the production of the photonnumber state with the lowest label in the expansion, $|1\rangle$ and $|2\rangle$, respectively. Larger values of $|\xi|$ motivate the increment of probabilities for other photon-number states; see Figure 4. 


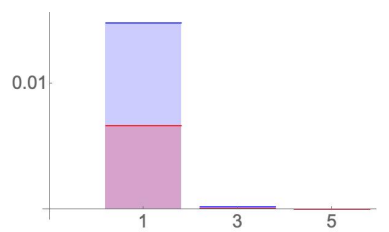

(a) $p=0$

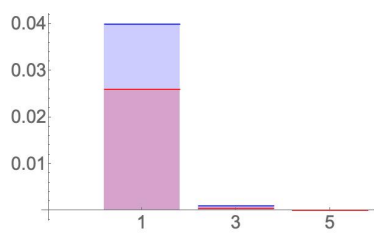

(b) $p=1$

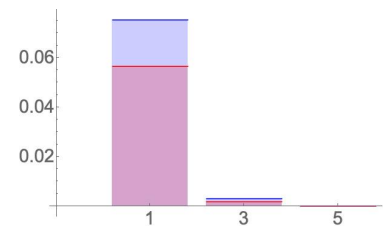

(c) $p=2$

Figure 2. Photon distribution for the $(2 p+1)$-subtracted squeezed-vacuum $\left|\xi_{2 p+1}^{\mathrm{vac}}\right\rangle$ (blue columns) and the $2 p$-subtracted odd-squeezed state $\left|\xi_{2 p}^{\text {odd }}\right\rangle$ (red columns). Both versions of subtracted states are composited by odd-photon number states only; see Table 1 . In all cases, $|\xi|=0.1$, and the value of $p$ is indicated in the caption.

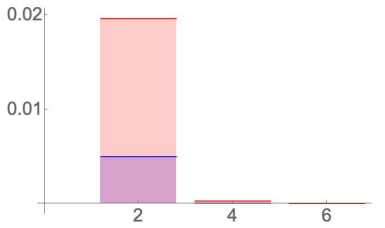

(a) $p=0$

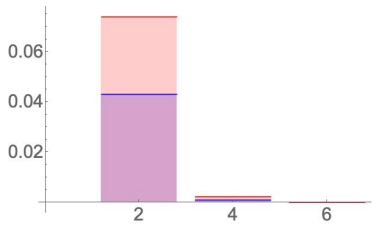

(b) $p=1$

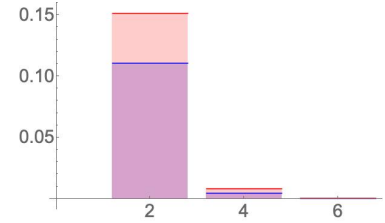

(c) $p=2$

Figure 3. Photon distribution for the $2 p$-subtracted squeezed-vacuum $\left|\xi_{2 p}^{\mathrm{vac}}\right\rangle$ (blue columns) and the $(2 p+1)$-subtracted odd-squeezed state $\left|\xi_{2 p+1}^{\text {odd }}\right\rangle$ (red columns). Both versions of subtracted states are composited by even-photon number states only; see Table 1 . In all cases, $|\xi|=0.1$, and the value of $p$ is indicated in the caption. Compare with Figure 2.

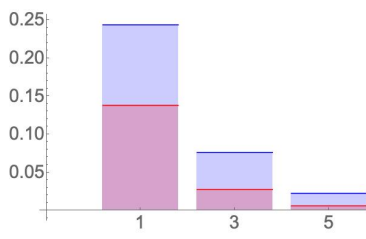

(a) $p=0$

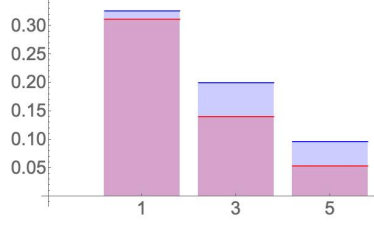

(b) $p=1$

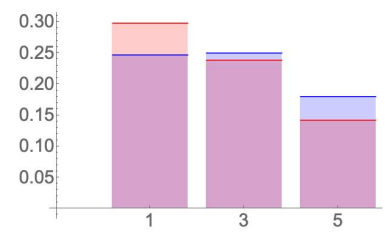

(c) $p=2$

Figure 4. Same as Figure 2, with $|\xi|=0.5$. Comparing with data in Figure 2, it is notable the increment of probabilities for photon-number states other than $|1\rangle$.

\subsection{Odd-Photon Squeezed States}

In a previous work [8], we have reported a new class of squeezed states that includes only odd-photon number states in their composition. These are called odd-photon squeezed states and are, in many respects, the counterpart of the squeezed-vacuum discussed in the previous section. Indeed, both the squeezed-vacuum and the odd-photon squeezed states satisfy the same second-order difference equation, although they are defined with different initial conditions [8].

To be concrete, the odd-photon squeezed states [8] are given by

$$
\left|\xi^{\text {odd }}\right\rangle=\frac{1}{\lambda^{\text {odd }}} \sum_{n=0}^{\infty} \frac{n !}{\sqrt{(2 n+1) !}}(-2 \xi)^{n}|2 n+1\rangle, \quad|\xi|<1,
$$

where

$$
\lambda^{\text {odd }}=\left[\frac{|\xi|}{\arcsin |\xi|}\right]^{-1 / 2}\left(1-|\xi|^{2}\right)^{-1 / 4}
$$

These vectors may be also written as [8]

$$
\left|\xi^{\text {odd }}\right\rangle=\frac{1}{\lambda^{\text {odd }} 1} F_{1}\left(\frac{1}{2}, \frac{3}{2}, \frac{1}{2} \xi \hat{a}^{\dagger 2}\right) \hat{S}(\xi)|1\rangle,
$$


with $\hat{S}(\xi)$ the squeezing operator (22). Thus, the odd-photon squeezed states $\left|\xi^{\text {odd }}\right\rangle$ are the result of applying a confluent hypergeometric-like version of the creation operator $\hat{a}^{\dagger}$ on the squeezed one-photon state $\hat{S}(\xi)|1\rangle$.

Applying the theory of photon subtraction to analyze the odd-photon squeezed state (24), we obtain the output $\left|\xi^{\text {odd }}, 0\right\rangle_{B}$ through Equation (16), with

$$
\left|\xi_{2 n}^{\text {odd }}\right\rangle=\frac{1}{\lambda_{2 n}^{\text {odd }}(\xi)} \sum_{k=0}^{\infty} \frac{(k+n) !}{\sqrt{(2 k+1) !}}(-2 \xi)^{k+n}|2 k+1\rangle
$$

and

$$
\left|\xi_{2 n+1}^{\text {odd }}\right\rangle=\frac{1}{\lambda_{2 n+1}^{\text {odd }}(\xi)} \sum_{k=0}^{\infty} \frac{(k+n) !}{\sqrt{(2 k) !}}(-2 \xi)^{k+n}|2 k\rangle .
$$

The normalizations are defined by the formulae

$$
\begin{aligned}
\left|\lambda_{2 n}^{\text {odd }}(\xi)\right|^{2} & =(2|\xi|)^{2 n}[\Gamma(n+1)]_{2}^{2} F_{1}\left(p+1, n+1, \frac{3}{2} ;|\xi|^{2}\right), \\
\left|\lambda_{2 n+1}^{\text {odd }}(\xi)\right|^{2} & =(2|\xi|)^{2 n}[\Gamma(n+1)]_{2}^{2} F_{1}\left(n+1, n+1, \frac{1}{2} ;|\xi|^{2}\right) .
\end{aligned}
$$

Figure 1 shows in continuous-red the probabilities to construct the $n$-subtracted states (27) and (28) by detecting $n$ photons in the idler mode. As in the previous example, no success $(n=0)$ is the most probable, event and the probability of success decays for $n>1$. Note, however, that the probability to get $\left|\xi_{1}^{\text {odd }}\right\rangle$ in the signal channel is relevant for almost any value of $|\xi|$. The reason is that this state is composited by even-photon number states $|2 n\rangle$, just as in $\left|\xi_{0}^{\mathrm{vac}}\right\rangle$. Depending on $n$, this similarity is observed for the other $n$-subtracted photon versions of $\left|\xi^{\mathrm{vac}}\right\rangle$ and $\left|\xi^{\text {odd }}\right\rangle$; see Table 1.

Table 1. The photon subtraction is available by injecting a light beam $|\psi\rangle$ into one of the input channels of a $50 / 50$ beam splitter $\hat{B}$, and the 0 -photon state $|0\rangle$ into the other. The output $|\Psi\rangle_{B}$ is a two-mode light beam that may exhibit nonclassical correlations between its modes, depending on the quantum properties of the input. Appropriate outputs $|\Psi\rangle$ lead to $n$-subtracted states $\left|\psi_{n}\right\rangle$ in one channel by measuring $n$ photons in the other channel. The squeezed-vacuum $\left|\xi^{\mathrm{vac}}\right\rangle$ and odd-photon squeezed $\left|\xi^{\text {odd }}\right\rangle$ states, entirely composited by even- and odd-photon number states, respectively, produce two-mode entangled light beams allowing photon subtraction. In both cases, detection of photons in one of the output channels may produce either even-like or odd-like $n$-subtracted beams at the other output channel.

\begin{tabular}{lcl}
\hline & $n$-Subtracted Photon States \\
\hline input & $\left|\xi^{\mathrm{vac}}\right\rangle$ & $\left|\xi^{\text {odd }}\right\rangle$ \\
even-like & $\left|\xi_{2 n}^{\mathrm{vac}}\right\rangle$ & $\left|\xi_{2 n+1}^{\text {odd }}\right\rangle$ \\
odd-like & $\left|\xi_{2 n+1}^{\text {vac }}\right\rangle$ & $\left|\xi_{2 n}^{\text {odd }}\right\rangle$ \\
\hline
\end{tabular}

The odd-squeezed states $\left|\xi^{\text {odd }}\right\rangle$ are also useful to produce single photons; see Figures 2-4. Indeed, Equation (24) may be rewritten in the form [8]

$$
\left|\xi^{\text {odd }}\right\rangle=\frac{1}{\lambda^{\text {odd }}} \sum_{n=0}^{\infty} \frac{n !}{(2 n+1) !}\left(-2 \xi a^{\dagger 2}\right)^{n}|1\rangle .
$$

Therefore, using the approximation $\lambda^{\text {odd }}\left|\xi^{\text {odd }}\right\rangle \approx|1\rangle-2 \sqrt{\frac{2}{3}} \xi|3\rangle$, we write

$$
\lambda^{\text {odd }}\left|\xi^{\text {odd }}, 0\right\rangle_{B} \approx|0\rangle_{a} \otimes\left(\frac{i}{\sqrt{2}}|1\rangle_{b}-\frac{1}{\sqrt{3}} \xi|3\rangle_{b}\right)+|1\rangle_{a} \otimes\left(\frac{1}{\sqrt{2}}|0\rangle_{b}-\xi|2\rangle_{b}\right)-2 \xi|2\rangle_{a} \otimes|1\rangle_{b}
$$


which predicts the production of one single photon in channel $b$ by detecting two photons in channel $a$, compare with (23). In contraposition with $\left|\xi^{\mathrm{vac}}\right\rangle$, the above expression shows that making $|\xi| \ll 1$ the series may be further truncated to get one-photon state $|1\rangle$ in the no success event.

On the other hand, controlling the $\xi$-parameter, Figure 5a shows the situation in which the one-photon state $|1\rangle$ occurs with the same probability for both $\left|\xi_{1}^{\text {vac }}\right\rangle$ and $\left|\xi_{0}^{\text {odd }}\right\rangle$. Note, however, the prevalence of the former over the latter to produce also states $|3\rangle$ and $|5\rangle$. That is, $\left|\xi_{0}^{\text {odd }}\right\rangle$ is more efficient to produce single photons $|1\rangle$ since it reduces the probabilities to get any other photon-number state. The roles are reversed if one pays attention to state $|2\rangle$; see Figure $5 \mathrm{~b}$. In this case, better than $\left|\xi_{1}^{\text {odd }}\right\rangle$, the state $\left|\xi_{0}^{\text {vac }}\right\rangle$ reduces the probabilities to get any other state $|2 n\rangle$.

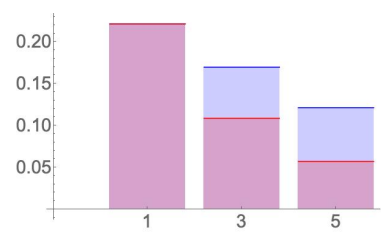

(a) odd-like

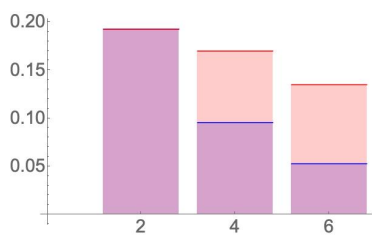

(b) even-like

Figure 5. Photon distribution for the $n$-subtracted states $\left|\xi_{1}^{\mathrm{vac}}\right\rangle,\left|\xi_{0}^{\text {odd }}\right\rangle$, with $|\xi|=0.783$ (a) and $\left|\xi_{0}^{\mathrm{vac}}\right\rangle,\left|\xi_{1}^{\text {odd }}\right\rangle$, with $|\xi|=0.813(\mathbf{b})$. The squeezing parameter has been selected to provide the same probability for states $|1\rangle$ and $|2\rangle,(\mathbf{a}, \mathbf{b})$, respectively.

Having two theoretical predictions to produce a given photon-number state, such as the ones shown in Figure 5, may serve to get a better fit between theoretical modeling and experimental data. By manipulating $\xi$, the theoretical photon distributions can be matched to the event of maximal counts, say this corresponds to the occurrence of $\left|n_{0}\right\rangle$. Then, comparing the theoretical predictions with the counts for other states $\left|n_{0} \pm k\right\rangle, n_{0} \geq k$, should determine whether $n$-subtracted version of $\left|\xi^{\text {vac }}\right\rangle$ and $\left|\xi^{\text {odd }}\right\rangle$ is the best option.

Figure 6 depicts the behavior of the Wigner distribution $W(z)$ [53] associated to the photon-subtracted states $\left|\xi_{n}^{\text {odd }}\right\rangle$ (details to construct this distribution in simple form can be consulted in Appendix A of reference [54]). The 0-photon subtracted case (Figure 6a) reproduces qualitatively the Wigner distribution of the odd-photon squeezed state $\left|\xi^{\text {odd }}\right\rangle$. The latter behaves as the distribution of the number state $|1\rangle$ when it is squeezed along one of the optical phase-space variables, where the amount of squeezing is steered by $|\xi|$. In general, the $2 n$-photon subtraction with $n>0$ induces the squeezing of the distribution by preserving the value of $|\xi|$ (Figures $6 \mathrm{~b}, \mathrm{c}$ ). After subtracting several photons, the distribution exhibits a series of oscillations, which is a signature of nonclassical behavior. In contrast, the subtraction of $(2 n+1)$ - photons changes the behavior of the distribution drastically. This is expected as the final distribution becomes a combination of even number states, and the corresponding Wigner distribution behaves in a similar way to that of squeezed vacuum states $\left|\xi^{\mathrm{vac}}\right\rangle$. However, negative regions appear even when a few photons are subtracted; see Figure 6d-f. 


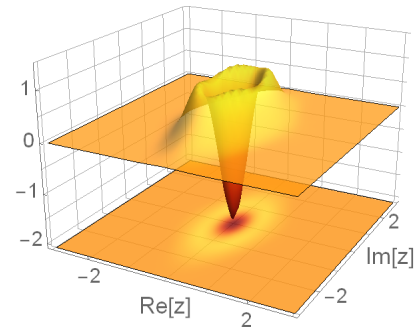

(a) $\left|\xi_{0}^{\text {odd }}\right\rangle$

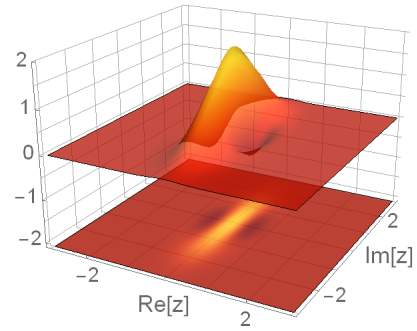

(d) $\left|\xi_{1}^{\text {odd }}\right\rangle$

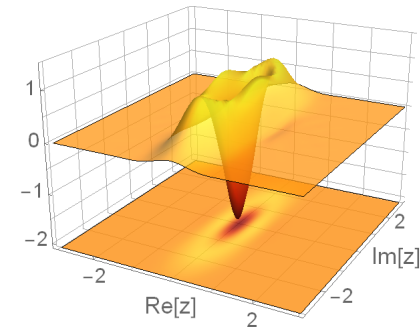

(b) $\left|\xi_{2}^{\text {odd }}\right\rangle$

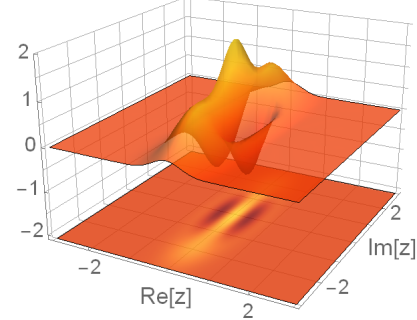

(e) $\left|\xi_{3}^{\text {odd }}\right\rangle$

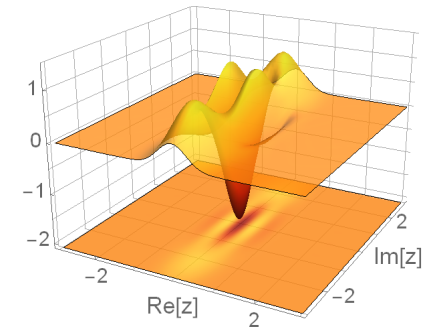

(c) $\left|\xi_{4}^{\text {odd }}\right\rangle$

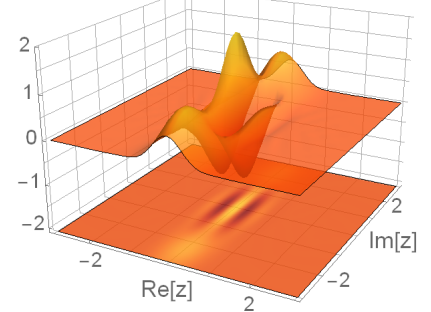

(f) $\left|\xi_{5}^{\text {odd }}\right\rangle$

Figure 6. Wigner distributions associated to the even-photon subtracted $\left|\xi_{2 n}^{\text {odd }}\right\rangle$ (first row) and oddphoton subtracted $\left|\xi_{2 n+1}^{\text {odd }}\right\rangle$ (second row) states generated out of the odd-photon squeezed states $\left|\xi^{o d d}\right\rangle$. The latter are depicted in terms of the optical phase-space variable $z=\operatorname{Re} z+\mathrm{i} \operatorname{Im} z$, with the squeezing parameter fixed as $|\xi|=0.5$ in all figures.

\subsection{Optical Cat States}

Optical cat states are defined as the quantum superposition of two opposite-phase Glauber states [9]:

$$
\begin{array}{r}
\left|z_{E}\right\rangle=\frac{e^{\frac{|z|^{2}}{2}}}{\lambda_{E}(z)}(|z\rangle+|-z\rangle)=\frac{1}{\lambda_{E}(z)} \sum_{n=0}^{\infty} \frac{z^{2 n}}{\sqrt{(2 n) !}}|2 n\rangle, \quad \lambda_{E}(z)=\sqrt{\cosh |z|^{2}}, \\
\left|z_{O}\right\rangle=\frac{e^{\frac{|z|^{2}}{2}}}{\lambda_{O}(z)}(|z\rangle+|-z\rangle)=\frac{1}{\lambda_{O}(z)} \sum_{n=0}^{\infty} \frac{z^{2 n+1}}{\sqrt{(2 n+1) !}}|2 n+1\rangle, \quad \lambda_{O}(z)=\sqrt{\sinh |z|^{2}} .
\end{array}
$$

These nonclassical states [52] have been successfully created in laboratory by subtracting photons from the squeezed-vacuum state [15-21].

Using $|0\rangle_{L}=|-z\rangle$ and $|1\rangle_{L}=|z\rangle$ as logical qubits [1], the optical cats are particularly useful for quantum information processing [15], where they are called Schrödinger kittens for $|z| \approx 1$.

The results in Section 4.1 apply immediately to analyze the related experimental data. As indicated above, the results in Section 4.2 represent a secondary option. Note, however, that the optical cat states (33) and (34) are formally different from both, the squeezedvacuum $\left|\xi^{\mathrm{vac}}\right\rangle$ and the odd-squeezed $\left|\xi^{\text {odd }}\right\rangle$ states. We have already mentioned that the main reason for such dissimilarity is that states $\left|z_{E}\right\rangle$ and $\left|z_{O}\right\rangle$, together with the Glauber states $|z\rangle$, belong to the space of solutions associated with a first-order difference equation. In turn, states $\left|\xi^{\mathrm{vac}}\right\rangle$ and $\left|\xi^{\text {odd }}\right\rangle$ are independent solutions of a second-order difference equation [8].

Within the photon subtraction scheme, the action of the beam splitter on the even cat $\left|z_{E}\right\rangle$ is easily calculated from Equation (15). Considering $\hat{a}|z\rangle=z|z\rangle$, one gets the entangled two-mode state

$$
\left|Z_{E}\right\rangle_{B}=\frac{1}{\sqrt{2}}\left[\left|\left(\frac{i z}{\sqrt{2}}\right)_{E}\right\rangle_{a} \otimes\left|\left(\frac{z}{\sqrt{2}}\right)_{E}\right\rangle_{b}+\left|\left(\frac{i z}{\sqrt{2}}\right)_{O}\right\rangle_{a} \otimes\left|\left(\frac{z}{\sqrt{2}}\right)_{O}\right\rangle_{b}\right] .
$$


For the odd cat $\left|z_{O}\right\rangle$, we use Equation (16), which yields

$$
\left|Z_{O}\right\rangle_{B}=\frac{1}{\sqrt{2}}\left[\left|\left(\frac{i z}{\sqrt{2}}\right)_{O}\right\rangle_{a} \otimes\left|\left(\frac{z}{\sqrt{2}}\right)_{E}\right\rangle_{b}+\left|\left(\frac{i z}{\sqrt{2}}\right)_{E}\right\rangle_{a} \otimes\left|\left(\frac{z}{\sqrt{2}}\right)_{O}\right\rangle_{b}\right] .
$$

Considering the logical qubits $|0\rangle_{L}=\left|z_{E}\right\rangle$ and $|1\rangle_{L}=\left|z_{O}\right\rangle$, we see that the above results are in the Bell-basis representation

$$
\left|Z_{E}\right\rangle_{B}=\frac{1}{\sqrt{2}}\left[|00\rangle_{L}+|11\rangle_{L}\right], \quad\left|Z_{O}\right\rangle_{B}=\frac{1}{\sqrt{2}}\left[|10\rangle_{L}+|01\rangle_{L}\right],
$$

so they represent two maximally entangled quantum states of a two-qubit bipartite system. The construction of Bell states as the result of passing optical cats by a beam splitter shows that the difference between the pairs $\left|Z_{E}\right\rangle,\left|Z_{O}\right\rangle$, and $\left|\xi^{\text {vac }}\right\rangle$, $\left|\xi^{\text {odd }}\right\rangle$, is not merely formal. In the latter case, entanglement is found between the elements of the superpositions defining the output modes, and not between the output superpositions by themselves.

In the language of quantum communication, the results of Equation (37) read as follows: If Alice (mode $a$ ) and Bob (mode $b$ ) measure their qubit then both of them find a random result, either $|0\rangle_{L}$ or $|1\rangle_{L}$ with probability $\frac{1}{2}$. Once Alice communicates her result to Bob (or vice versa), they find that their results are perfectly correlated, although their own outcomes seemed random. In the present case, Alice does not require to 'read' her qubit entirely. She needs to count the related number of photons only. In the even case, if she finds an even number of photons, then Bob will find an even number of photons, with certainty. Similarly, if she finds an odd number of photons, then Bob will read odd photons. A combined lecture of the number of photons is achieved in the odd case, since Alice will read an odd number of photons, while Bob counts an even number, and vice versa.

\section{Conclusions}

We have studied how a two-mode entangled light beam can be produced by injecting superpositions of photon-number states into a lossless symmetric beam splitter. As the process changes the quantum statistics of the input fields, nonclassical correlations are stimulated between the output modes whenever the incident beam is nonclassical. We have considered the expansion of the output field in terms of the two-mode representation of $S U(2)$ coherent states, which encode nonclassical correlations between their modes. We derived a compact expression for the output field that is useful to construct photon subtracted versions of any input superposition of photon-number states. As applications, we derived the analytic form that links the detection of $n$-photons in the idler channel with the $n$-subtracted version of the squeezed-vacuum state in the signal channel. A second example provided the $n$-photon subtracted form of the odd-vacuum states, which represents a new class of nonclassical states. We have also shown that photon subtraction of classical fields does not produce nonclassical fields, as well as that injecting optical cats into a beam splitter produces entangled states in the Bell-basis representation.

Author Contributions: All authors contributed equally to this work. All authors have read and agreed to the published version of the manuscript.

Funding: This research has been funded by Consejo Nacional de Ciencia y Tecnología (CONA- CyT), Mexico, Grant Numbers A1-S-24569 and CF19-304307.

Institutional Review Board Statement: Not applicable.

Informed Consent Statement: Not applicable.

Data Availability Statement: Not applicable.

Acknowledgments: K. Zelaya acknowledges the support from the project "Physicists on the move II" (KINEÓ II), Czech Republic, Grant No. CZ.02.2.69/0.0/0.0/18053/0017163.

Conflicts of Interest: The authors declare no conflict of interest. 


\section{Appendix A. Useful Material}

The first basis elements (3) are as follows:

$$
\begin{aligned}
& |0,0\rangle_{B}=|0,0\rangle, \quad|1,0\rangle_{B}=\frac{1}{\sqrt{2}}(|0,1\rangle+i|1,0\rangle), \\
& |2,0\rangle_{B}=\frac{1}{2}(|0,2\rangle+i \sqrt{2}|1,1\rangle-|2,0\rangle) \\
& |3,0\rangle_{B}=\frac{1}{2 \sqrt{2}}(|0,3\rangle+i \sqrt{3}|1,2\rangle-\sqrt{3}|2,1\rangle-i|3,0\rangle) .
\end{aligned}
$$

These vectors encode nonclassical correlations between modes $a$ and $b$. For instance, $|1,0\rangle_{B}$ in (A1) is one of the elements in the Bell basis, which is prototypical to describe entanglement in bipartite qubit systems.

The two-mode state (5) can be expressed as follows:

$$
|\Psi\rangle_{B}=\frac{1}{\lambda} \sum_{n, k=0}^{\infty} \alpha_{k+n} c_{n, k}|n\rangle_{a} \otimes|k\rangle_{b}
$$

where

$$
c_{n, k}=\frac{1}{\sqrt{2^{n+k}}}\left(\begin{array}{c}
n+k \\
n
\end{array}\right)^{1 / 2} e^{i \frac{\pi}{2} n}
$$

Then,

$$
|\Psi\rangle_{B}=\frac{1}{\lambda} \sum_{n, k=0}^{\infty} \frac{\sqrt{(k+n) !}}{\sqrt{n !} \sqrt{k !}} e^{i \frac{\pi}{2} n} \beta_{k+n}|n\rangle_{a} \otimes|k\rangle_{b}, \quad \beta_{n}=\frac{\alpha_{n}}{\sqrt{2^{n}}} .
$$

Using Equation (9), we have either

$$
|\Psi\rangle_{B}=\frac{1}{\lambda} \sum_{n=0}^{\infty} \frac{e^{i \frac{\pi}{2} n}}{\sqrt{n !}} \lambda_{n}(\beta)|n\rangle_{a} \otimes\left|\psi_{n}(\beta)\right\rangle_{b},
$$

or

$$
|\Psi\rangle_{B}=\frac{1}{\lambda(\alpha)} \sum_{k=0}^{\infty} \frac{e^{-i \frac{\pi}{2} k}}{\sqrt{k !}} \lambda_{k}(\beta)\left|\psi_{k}(\beta)\right\rangle_{a} \otimes|k\rangle_{b} .
$$

These two expressions are equivalent.

For input states expressed as linear superpositions consisting entirely of even-photon states

$$
\left|\psi_{E}\right\rangle=\frac{1}{\lambda_{E}} \sum_{n=0}^{\infty} \alpha_{2 n}|2 n\rangle,
$$

we have

$$
\begin{aligned}
\left|\Psi_{E}\right\rangle_{B}=\frac{1}{\lambda_{E}} \sum_{n, k=0}^{\infty}\left[\alpha_{2(k+n)} c_{2 n, 2 k}|2 n\rangle_{a} \otimes|2 k\rangle_{b}\right. & \\
& \left.+\alpha_{2(k+n+1)} c_{2 n+1,2 k+1}|2 n+1\rangle_{a} \otimes|2 k+1\rangle_{b}\right] .
\end{aligned}
$$

Using (A3) and (9) yields

$$
\begin{aligned}
\left|\Psi_{E}\right\rangle_{B}=\frac{1}{\lambda_{E}} \sum_{n=0}^{\infty}\left[\frac{e^{i \pi n}}{\sqrt{(2 n) !}} \lambda_{2 n}^{E}(\beta)|2 n\rangle_{a} \otimes\left|\psi_{2 n}^{E}(\beta)\right\rangle_{b}\right. & \\
& \left.+\frac{i e^{i \pi n}}{\sqrt{(2 n+1) !}} \lambda_{2 n+1}^{E}(\beta)|2 n+1\rangle_{a} \otimes\left|\psi_{2 n+1}^{E}(\beta)\right\rangle_{b}\right],
\end{aligned}
$$

where

$$
\left|\psi_{2 n}^{E}(\alpha)\right\rangle_{b}=\frac{1}{\lambda_{2 n}^{E}(\alpha)} \sum_{k=0}^{\infty} \sqrt{\frac{(2 k+2 n) !}{(2 k) !}} \alpha_{2(k+n)}|2 k\rangle_{b}
$$


and

$$
\left|\psi_{2 n+1}^{E}(\alpha)\right\rangle_{b}=\frac{1}{\lambda_{2 n+1}^{O}(\alpha)} \sum_{k=0}^{\infty} \sqrt{\frac{(2 k+2 n+2) !}{(2 k+1) !}} \alpha_{2(k+n+1)}|2 k+1\rangle_{b} .
$$

The case of superpositions including only odd-photon states

$$
\left|\psi_{O}\right\rangle=\frac{1}{\lambda_{O}} \sum_{n=0}^{\infty} \alpha_{2 n+1}|2 n+1\rangle
$$

gives the result

$$
\begin{aligned}
\left|\Psi_{O}\right\rangle_{B}=\frac{1}{\lambda_{O}} \sum_{n, k=0}^{\infty}\left[\alpha_{2(k+n)+1} c_{2 n, 2 k+1}|2 n\rangle_{a} \otimes|2 k+1\rangle_{b}\right. & \\
& \left.+\alpha_{2(k+n)+1} c_{2 n+1,2 k}|2 n+1\rangle_{a} \otimes|2 k\rangle_{b}\right],
\end{aligned}
$$

which is simplified as follows:

$$
\begin{aligned}
\left|\Psi_{O}\right\rangle_{B}=\frac{1}{\lambda_{O}} \sum_{n=0}^{\infty}\left[\frac{e^{i \pi n}}{\sqrt{(2 n) !}} \lambda_{2 n}^{O}(\beta)|2 n\rangle_{a} \otimes\left|\psi_{2 n}^{O}(\beta)\right\rangle_{b}\right. \\
\left.+\frac{i e^{i \pi n}}{\sqrt{(2 n+1) !}} \lambda_{2 n+1}^{O}(\beta)|2 n+1\rangle_{a} \otimes\left|\psi_{2 n+1}^{O}(\beta)\right\rangle_{b}\right],
\end{aligned}
$$

where

$$
\left|\psi_{2 n}^{O}(\alpha)\right\rangle_{b}=\frac{1}{\lambda_{2 n}^{O}(\alpha)} \sum_{k=0}^{\infty} \sqrt{\frac{(2 k+2 n+1) !}{(2 k+1) !}} \alpha_{2(k+n)+1}|2 k+1\rangle_{b}
$$

and

$$
\left|\psi_{2 n+1}^{O}(\alpha)\right\rangle_{b}=\frac{1}{\lambda_{2 n+1}^{O}(\alpha)} \sum_{k=0}^{\infty} \sqrt{\frac{(2 k+2 n+1) !}{(2 k) !}} \alpha_{2(k+n)+1}|2 k\rangle_{b} .
$$

\section{References}

1. O’Brien, J.L.; Furusawa, A.; Vužović, J. Photonic quantum technologies. Nat. Photonics 2009, 3, 687. [CrossRef]

2. Gisin, N.; Thew, R. Quantum Communication. Nat. Photonics 2007, 1, 165. [CrossRef]

3. Knill, E.; Laflamme, R.; Milburn, G.J. A scheme for efficient quantum computation with linear optics. Nature 2001, $409,46$. [CrossRef] [PubMed]

4. Walls, D.F. Squeezed states of light. Nature 1983, 306, 141. [CrossRef]

5. Rosas-Ortiz, O. Coherent and squeezed states: Introductory review of basic notions, properties and generalizations. In Integrability, Supersymmetry and Coherent States; Kuru, S., Negro, J., Nieto, L.M., Eds.; CRM Series in Mathematical Physics; Springer: Cham, Switzerland, 2019.

6. Barrios-Barocio, E.; Velázquez, V.; Cruz, S.C.Y. Design and Construction of Homodyne Detectors for the Study of Quantum Optical States. J. Phys. Conf. Ser. 2020, 1540, 012030.

7. Lvovsky, A.I. Squeezed light. In Photonics Volume 1: Fundamentals of Photonics and Physics; Andrews, D., Ed.; Wiley: Hoboken, NJ, USA, 2015; pp. 121-164.

8. Zelaya, K.; Hussin, V.; Rosas-Ortiz, O. Constructing Squeezed States of Light with Associated Hermite Polynomials. Eur. Phys. J. Plus 2021, 136, 534. [CrossRef]

9. Dodonov, V.V.; Malkin, I.A.; Man'ko, V.I. Even and odd coherent states and excitations of a singular oscillator. Physica 1974, 72, 597. [CrossRef]

10. Glauber, R.J. Quantum Theory of Optical Coherence: Selected Papers and Lectures; Wiley-VCH: Weinheim, Germany, 2007.

11. Schrödinger, E. Die gegenwärtige Situation in der Quantenmechanik. Die Naturwissenschaften 1935, 23, 807-812.

12. Agarwal, G.S. Quantum Optics; Cambridge University Press: Cambridge, UK, 2013

13. Bachor, H.; Ralph, T.C. A Guide to Experiments in Quantum Optics, 3rd ed.; Wyley-VCH: Weinheim, Germany, 2019.

14. Zhang, M.; Kang, H.; Wang, M.; Xu, F.; Su, X.; Peng, K. Quantifying quantum coherence of optical cat states. Photonics Res. 2021, 9, 887. [CrossRef]

15. Ourjoumtsev, A.; Tualle-Brouri, R.; Laurat, J.; Grangier, P. Generating Optical Schrödinger Kittens for Quantum Information Processing. Science 2006, 312, 83. 
16. Dakna, M.; Anhut, T.; Opartny, T.; Knoll, L.; Welsch, D.-G. Generating Schrödinger-cat-like states by means of conditional measurements on a beam splitter. Phys. Rev. A 1997, 55, 3184.

17. Neergaard-Nielsen, J.S.; Nielsen, B.M.; Hettich, C.; Mølmer, K. Generation of a superposition of odd photon number states for quantum information networks. Phys. Rev. Lett. 2006, 97, 083604. [CrossRef]

18. Wakui, K.; Takahashi, H.; Furusawa, A.; Sasaki, M. Photon subtracted squeezed states generated with periodically poled KTiOPO 4 . Opt. Express 2007, 15, 3568. [PubMed]

19. Gerrits, T.; Glancy, S.; Clement, T.S.; Calkins, B.; Lita, A.E.; Miller, A.J.; Migdall, A.L.; Nam, S.W.; Mirin, R.P.; Knill, E. Generation of optical coherent-state superpositions by number-resolved photon subtraction from the squeezed vacuum. Phys. Rev. A 2010, 82, 031802. [CrossRef]

20. Namekata, N.; Takahashi, Y.; Fukii, G.; Fukuda, D.; Kurimura, S.; Inoue, S. Non-Gaussian operation based on photon subtraction using a photon-number-resolving detector at a telecommunications wavelength. Nat. Photonics 2010, 4, 655. [CrossRef]

21. Asavanant, W.; Nakashima, K.; Shiozawa, Y.; Yoshikawa, J.; Furusawa, A. Generation of highly pure Schrödinger's cat states and real-time quadrature measurements via optical filtering. Opt. Express 2017, 25, 32227. [CrossRef]

22. Ralph, T.C.; Gilchrist, A.; Milburn, G.J.; Munro, W.J.; Glancy, S. Quantum computation with optical coherent states. Phys. Rev. A 2003, 68, 042319.

23. Van Enk, S.J.; Hirota, O. Entangled coherent states: Teleportation and decoherence. Phys. Rev. A 2001, 64, 022313. [CrossRef]

24. Kim, M.S.; Park, E.; Knight, P.L.; Jeong, H. Nonclassicality of a photon-subtracted Gaussian field. Phys. Rev. A 2005, 71, 043805. [CrossRef]

25. Biswas, A.; Agarwal, G.S. Nonclassicality and decoherence of photon-subtracted squeezed states. Phys. Rev. A 2007, 75, 032104.

26. Clausen, J.; Knoll, L.; Welsch, D.G. Lossy purification and detection of entangled coherent states. Phys. Rev. A 2002, 66, 062303. [CrossRef]

27. Sheng, Y.B.; Zhou, L.; Long, G.L. Hybrid entanglement purification for quantum repeaters. Phys. Rev. A 2013, 88, 022302. [CrossRef]

28. Zhang, L.M.; Gao, T.; Yan, F.L. Transformations of multilevel coherent states under coherence-preserving operations. Sci. China Phys. Mech. Astron. 2021, 64, 260312. [CrossRef]

29. Yan, P.S.; Zhou, L.; Zhong, W.; Sheng, Y.B. Measurement-based entanglement purification for entangled coherent states. Front. Phys. 2021, 17, 21501. [CrossRef]

30. Wenger, J.; Tualle-Brouri, R.; Grangier, P. Non-Gaussian statistics from individual pulses of squeezed light. Phys. Rev. Lett. 2004, 92, 153601. [CrossRef]

31. Olivares, S.; Paris, M.G.A. Squeezed Fock state inconclusive photon subtraction. J. Opt. B Quantum Semiclass. Opt. 2005, 7, S616. [CrossRef]

32. Sanders, B.C. Quantum dynamics of the nonlinear rotator and the effects of continual spin measurement. Phys. Rev. A 1989, 40, 2417. [CrossRef] [PubMed]

33. Boto, A.N.; Kok, P.; Abrams, D.S. Quantum Interferometric Optical Lithography: Exploiting Entanglement to Beat the Diffraction Limit. Phys. Rev. Lett. 2000, 85, 2733. [CrossRef] [PubMed]

34. Kok, P.; Boto, A.N.; Abrams, D.S.; Williams, C.P.; Braunstein, S.L.; Dowling, J.P. Quantum interferometric optical lithography: Towards arbitrary two-dimensional patterns. Phys. Rev. A 2001, 63, 063407. [CrossRef]

35. Lee, H.; Kok, P.; Dowling, J.P. A quantum Rosetta stone for interferometry. J. Mod. Opt. 2002, 49, 2325. [CrossRef]

36. Kok, P.; Lee, H.; Dowling, J.P. Creation of large-photon-number path entanglement conditioned on photodetection. Phys. Rev. A 2002, 65, 052104. [CrossRef]

37. Hong, C.K.; Mandel, L. Theory of parametric frequency down conversion of light. Phys. Rev. A 1985, 31, 2409. [CrossRef]

38. Hong, C.K.; Mandel, L. Measurement of subpicosecond time intervals between two photons by interference. Phys. Rev. Lett. 1987, 59, 2044. [PubMed]

39. Pryde, G.J.; White, A.G. Creation of maximally entangled photon-number states using optical fiber multiports. Phy. Rev. A 2003, 68, 052315.

40. Walther, P.; Pan, J.; Aspelmeyer, M.; Ursin, R.; Gasparoni, S.; Zeilinger, A. De Broglie wavelength of a non-local four-photon state. Nature 2004, 429, 158. [PubMed]

41. Afek, I.; Ambar, O.; Silberberg, Y. High-NOON States by Mixing Quantum and Classical Light. Science 2010, 328, 879. [PubMed]

42. Israel, Y.; Afek, I.; Rosen, S.; Silberberg, Y. Experimental tomography of NOON states with large photon numbers. Phys. Rev. A 2012, 85, 022115.

43. Campos, R.A.; Saleh, B.E.A.; Teich, M.C. Quantum-mechanical lossless beam splitter: SU(2) symmetry and photon statistics. Phys. Rev. A 1989, 40, 1371.

44. Perelomov, A. Generalized Coherent States and Their Applications; Springer: Berlin, Germany, 1986.

45. Moran, J.; Hussin, V. A New Method for Constructing Squeezed States for the Isotropic 2D Harmonic Oscillator. In Quantum Theory and Symmetries; Paranjape, M.B., MacKenzie, R., Thomova, Z., Winternitz, P., Witczak-Krempa, W., Eds.; CRM Series in Mathematical Physics; Springer: Cham, Switzerland, 2021.

46. Bužek, V.; Quang, T. Generalized coherent state for bosonic realization of SU(2) Lie algebra. J. Opt. Soc. Am. B 1989, 6, 2447.

47. Wodkiewicz, K.; Eberly, J.H. Coherent states, squeezed fluctuations, and the $S U(2)$ am $S U(1,1)$ groups in quantum-optics applications. J. Opt. Soc. Am. B 1985, 2, 458. 
48. Kim, M.S.; Son, W.; Bužek, V.; Knight, P.L. Entanglement by a beam splitter: Nonclassicality as a prerequisite for entanglement. Phys. Rev. A 2002, 65, 032323. [CrossRef]

49. Lai, W.K.; Bužek, V.; Knight, P.L. Nonclassical fields in a linear directional coupler. Phys. Rev. A 1991, 43, 6323. [CrossRef] [PubMed]

50. Olver, F.W.; Lozier, D.W.; Boisvert, R.F.; Clark, C.W. (Eds.) NIST Handbook of Mathematical Functions; Cambridge University Press: Cambridge, UK, 2010.

51. Zelaya, K.; Rosas-Ortiz, O.; Blanco-Garcia, Z.; Cruz, S.C.Y. Completeness and Nonclassicality of Coherent States for Generalized Oscillator Algebras. Adv. Math. Phys. 2017, 2017, 7168592. [CrossRef]

52. Dodonov, V.V.; Man'ko, V.I. (Eds.) Theory of Nonclassical States of Light; Taylor and Francis: New York, NY, USA, 2003.

53. Ulf, L. Measuring the Quantum State of Light; Cambridge University Press: Cambridge, UK, 1997.

54. Zelaya, K.; Dey, S.; Hussin, V. Generalized squeezed states. Phys. Lett. A 2018, 382, 3369. [CrossRef] 